presence of very small amounts of Ia antigens on less than $50 \%$ of thymocytes, a finding made possible by the use of a fluorescence-activated electronic cell sorter (Fathman et al., J. Immun., 115, 584; 1975). More conventional procedures such as immunofluorescence, cytotoxicity, and absorption of antisera, do not allow detection of Ia antigens on thymocytes although they readily do so on B cells.

The presence of small amounts of Ia antigens on thymocytes is more than vaguely similar to the story of $\mathrm{Ig}$ on thymocytes and $T$ cells. Since it seems that the presence of $\mathrm{Ig}$ on $\mathrm{T}$ cells is sometimes a result of its passive adsorption on to the surfaces of the cells, and not to its endogenous synthesis, an obvious point arises as to whether the same is true for Ia antigens and thymocytes. Indeed, it has been demonstrated by Vitetta et al. (J. Immunogenet., 1,$82 ; 1974)$ that, in contrast to $\mathrm{H} 2$ antigens, soluble Ia antigens are readily shed into incubation medium containing Ia-positive lymphocytes. In addition, passage of Ia-positive lymphocytes down a column of nylon wool (a procedure used to deplete cell populations of $\mathbf{B}$ cells) seems to remove selectively some of the Ia antigens from the surface of the B cells. (Schultz et al., Cell. Immun., 16, 125; 1975, and Fathman et al., J. Immun., 115, 584; 1975).

These intriguing findings, besides being of some significance themselves with respect to Ia antigen function, will no doubt ensure perpetuation of the controversy surrounding the presence and relationship of $I a$ antigens to $T$ cells.

\section{More new particles}

In the article 'More new particles' which appeared in News and Views last week an editorial change implied that a section was a full account of work discussed at the recent Lepton-Photon conference at Stanford; the article was in fact received before the Stanford conference.

\section{Nuclear states of high spin}

\section{from P. E. Hodgson}

ONE of the most powerful ways of determing the spins of nuclear states is by one-nucleon transfer reactions: the angular distribution of the emitted particles is characteristic of the angular momentum transfer, and this usually suffices, sometimes in association with other data, to fix the spin of the final state in the residual nucleus.

This method works best for low spins, corresponding to angular momentum transfers of 0,1 and 2 . For higher spins the angular distribution is not so characteristic, and the reaction may be forbidden by the spin selection rules.

Among the methods applicable to high spin states the $\left({ }^{7} \mathrm{Li}, \mathrm{p}\right)$ reaction is proving useful, and a recent paper by Bishop and Fortune (Phys. Rev. Lett., 34, 1350; 1975) provides a good illustration of this.

At low energies on light nuclei the $\left({ }^{7} \mathrm{Li}, \mathrm{p}\right)$ reaction proceeds predominantly through the compound nucleus. The ${ }^{7} \mathrm{Li}$ is captured by the target nucleus to form a compound system which then can decay by proton emission. It is then found that the total cross section for the reaction is closely proportional to $(2 J+1)$, where $J$ is the spin of the final nuclear state. This relation has been tested for a large number of states, and can be used with some confidence to determine unknown spins.

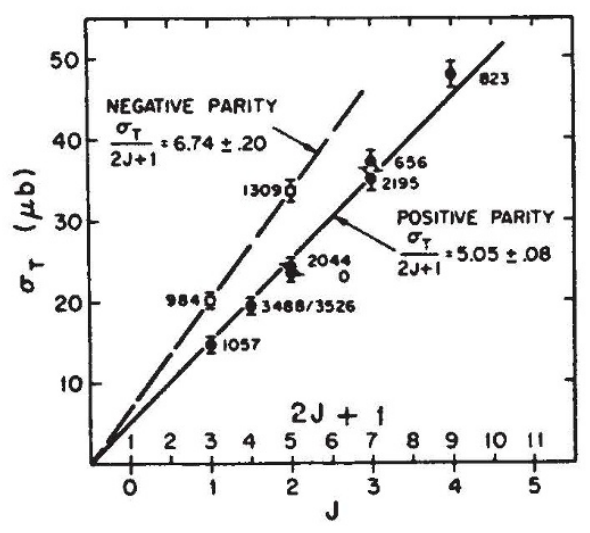

Total cross sections for the ${ }^{14} \mathrm{~N}\left({ }^{7} \mathrm{Li}, \mathrm{p}\right)$ ${ }^{20} \mathrm{~F}$ reaction to a number of positive and negative parity states of ${ }^{20} \mathrm{~F}$ plotted as a function of $(2 J+1)$.

Bishop and Fortune studied the reaction ${ }^{14} \mathrm{~N}\left({ }^{7} \mathrm{Li}, \mathrm{p}\right){ }^{20} \mathrm{~F}$, and their results for some positive and negative parity states of ${ }^{20} \mathrm{~F}$ with known spins are shown in the figure. The cross sections are closely proportional to $(2 J+1)$ and the ratio of the total cross section to $(2 J+1)$ is $5.05 \pm 0.08$ for the positive parity states and $6.74 \pm 0.20$ for the negative parity states. The proportionality is so closely followed that it is possible to use the total cross sections for the $\left({ }^{7} \mathrm{Li}, \mathrm{p}\right)$ reaction to determine the spins of some other states. The identification can be strengthened by seeing how well the state fits into the rotational band structure of the lowlying states of ${ }^{20} \mathrm{~F}$.

The method used is simply to calculate the spin from the expression $\frac{1}{2}\left[\left(\sigma_{\mathrm{T}} / 5.05\right)-1\right]$ for positive parity states and $\frac{1}{2}\left[\left(\sigma_{\mathrm{T}} / 6.74\right)-1\right]$ for negative parity states and to compare these spin values with those of the possible states that fit into the band structure.

For the state at $2.87 \mathrm{MeV}$ for example, the calculated spins are 3.5 for a positive parity state and 2.5 for negative parity. For the known band structure the assignment $3^{-}$is considered the most likely. In a similar way one of two states at $2.97 \mathrm{MeV}$ is probably identified as $4^{-}$. Probable spin assignments are made to several other states as well.

This work shows the usefulness of the $\left({ }^{7} \mathrm{Li}, \mathrm{p}\right)$ reaction in determining the spins of nuclear states, when used in conjunction with other techniques.

\section{Bird song dialects}

\section{from John R. Krebs}

Song birds, like humans, have local dialects. Regional variations in the form of territorial song have been found in such birds as chaffinches, treecreepers, cardinals, white-crowned sparrows and song sparrows. Often dialect regions are quite small, in the order of a few miles across, and the transition between them may be very sudden. Within a dialect region all males have similar songs whereas between regions the song varies considerably. Playback experiments in which recorded songs are broadcast inside a territory have shown that males can tell the difference between songs of their own dialect and those from other regions (Lemon, Anim. Behav., 15, 538-595; 1967; Milligan and Verner, Condor, 73, 208-213; 1971); territorial males react more vigorously to songs of their own dialect region.

Dialects probably originate as a result of young birds learning the songs of nearby adults soon after leaving the nest. In the white-crowned sparrow, for example, young males learn the territorial song within the first three months after leaving the nest, so that they are likely to learn the characteristic song of their birthplace.

Several workers have suggested that dialects play a role in the genetic adaptation of populations to local environmental conditions, by acting as a reproductive barrier between populations. This is based on the assumption, as yet untested, that females as well as males can recognise songs of their own dialect region, and will mate preferentially with local birds. Nottebohm (Condor, 71, 299-315; 1969) found that dialect boundaries in a South American sparrow, the chingolo, correspond with changes in habitat structure, for example from grassland to forest, which is what one would expect if dialect regions represented locally 